\title{
The genetic structure of Parus caeruleus (blue tit) populations as revealed by minisatellite single locus probes
}

\author{
G. R. VERHEYEN $\dagger^{*}$, B. KEMPENAERS $\dagger \ddagger$, F. ADRIAENSEN $\dagger$, M. VAN DEN BROECK $\S$, \\ E. MATTHYSEN $\dagger$, C. VAN BROECKHOVEN§ \& A. A. DHONDT† \\ †Laboratory of Animal Ecology, Department of Biology and \$Laboratory of Neurogenetics, Department of \\ Biochemistry, University of Antwerp (UIA), Universiteitsplein 1, 2610 Wilrijk, Belgium
}

\begin{abstract}
The genetic structure of three blue tit (Parus caeruleus) populations near Antwerp in Belgium was determined with the use of three hypervariable minisatellite DNA single locus probes. The degree of genetic variability within and among populations, deviations from Hardy-Weinberg equilibrium and population differentiation were estimated to determine whether these DNA regions are informative for studying population processes in birds. All populations were significantly differentiated. In one population, consisting of several subpopulations in discrete woodlots, a slight heterozygote deficit (Wahlund effect) was observed. More detailed information concerning the population structure was found using Wright's $F$-statistics. Gene flow between all populations was high. Furthermore, Nei's genetic distance showed a slight increase with increase of geographical distance between, populations, despite their close proximity.
\end{abstract}

Keywords: blue tit, $F$-statistics, genetic distance, minisatellite, population structure, single locus probe.

\section{Introduction}

Several studies have used genetic markers to study the structure of bird populations. Most investigations have been based on protein and mitochondrial DNA polymorphisms (Rockwell \& Barrowclough 1987; Ball et al. 1988; Johnson \& Marten 1989; Moore et al., 1991; Taberlet et al., 1992; Wenink et al., 1993). Most indicate that gene flow is high, and therefore genetic differences are low, between bird populations. However, some studies show higher levels of differentiation between populations (Leberg, 1990; Stangel et al., 1992).

The DNA fingerprinting technique was developed in 1985 by Jeffreys et al. Multilocus probes are used to detect several hypervariable minisatellite (VNTR) loci at the same time, thus yielding a very complex and individual-specific banding pattern or DNA fingerprint. Consequently, this technique has found widespread use in several fields including individual identification in forensics (Jeffreys et al., 1985),

\section{*Correspondence.}

¿Present address: Department of Biology, Queen's University, Kingston, Ontario, Canada K7L 3N6. paternity analysis in human and animal species (Jeffreys et al., 1986; Burke \& Bruford, 1987) and determination of population structure (Gilbert et al., 1990; Haig et al., 1993). Because of the impossibility of identifying the alleles of individual loci in this highly complex banding pattern, DNA fingerprints are difficult to compare between gels. This renders the technique less useful in studies in which it is desirable to characterize the alleles of separate loci. In such studies, hypervariable single locus probes (SLPs), detecting only one minisatellite locus, can be useful. Unfortunately, most of these markers are species-specific and thus have to be developed for each species separately (Hanotte et al., 1991).

We have used DNA fingerprinting in order to assign extra-pair paternity in the blue tit (Parus caeruleus: Kempenaers et al., 1992, 1995), a small forestdwelling passerine bird (Dhondt, 1989). For this purpose, several hypervariable minisatellite SLPs were developed (Verheyen et al., 1994).

In this paper we present results on the application of this relatively new type of genetic marker (three hypervariable minisatellite SLPs) to describe the genetic population structure of blue tits in a number of forest fragments around Antwerp, North Belgium. 
The markers are believed to be selectively neutral (Clark 1987; Jeffreys et al., 1988), which makes them potentially very useful for these kinds of studies. It can thus be assumed that differences between populations at these loci are most probably caused by migration and random genetic drift and not by selection.

\section{Materials and methods}

\section{Study areas}

Blue tits were sampled from three populations near Antwerp: plot Boswachter (plot B in Dhondt (1989), $12.5 \mathrm{ha}$ ) is part of the large 'mainland' population Peerdsbos (total $>100$ ha, hereafter called PB), Calixbergen (hereafter called CX, plot $\mathrm{C}$ in Dhondt (1989), $17 \mathrm{ha}$ ) is an isolated estate situated at about $2 \mathrm{~km}$ south of the Peerdsbos, and the Boshoek population (hereafter called BO) comprises four subpopulations in discrete woodlots (VS, 1.5 ha and LA, 7.3 ha, which were always combined because of low sample sizes, $\mathrm{KB} 11.8$ ha and $\mathrm{ZZ} 11.5$ ha; all subpopulations are within 0.5 to $2 \mathrm{~km}$ of each other). The Boshoek population is situated about 13 $\mathrm{km}$ south of Calixbergen. The populations are situated in a landscape matrix of residential and agricultural areas.

In all study areas, small-holed nestboxes in which blue tits can breed, have been present for several years. Despite some differences, all areas can be considered to be suitable habitats for blue tits.

\section{Blood samples and DNA extraction}

During spring, blood samples of $10-50 \mu \mathrm{L}$ were collected in the field by venipuncture of the brachial vein of captured adult blue tits and were stored at $-70^{\circ} \mathrm{C}$. After thawing, $10 \mu \mathrm{L}$ of whole blood was suspended in $600 \mu \mathrm{L} 1 \times$ SET buffer $(0.15 \mathrm{M} \mathrm{NaCl}, 1$ mM EDTA, $50 \mathrm{~mm}$ Tris- $\mathrm{HCl}, \mathrm{pH} 8.0$ ). The mixture was incubated overnight at $55^{\circ} \mathrm{C}$ in the presence of $1.5 \mathrm{U} / \mathrm{mL}$ proteinase $\mathrm{K}$ and 0.3 per cent SDS. DNA was extracted once with one volume of phenol and twice with one volume of phenol/chloroform/isoamylalcohol (25/24/1). The DNA was precipitated with 99 per cent ethanol and resuspended in $50 \mu \mathrm{L}$ $1 \times \mathrm{TE}^{-4}$ buffer ( $1 \mathrm{~mm}$ Tris- $\mathrm{HCl}, 0.01 \mathrm{~mm}$ EDTA, $\mathrm{pH} 7.5)$.

\section{Single locus probes}

Three blue tit specific SLPs (cPcaMS1, cPcaMS3 and cPcaMS14, hereafter called MS1, MS3 and
MS14, respectively) were used in this study. Details of the isolation and characterization of these minisatellite markers are described in Verheyen et al. (1994).

\section{Electrophoresis, radiolabelling and hybridization}

Four $\mu \mathrm{g}$ of DNA was digested overnight at $37^{\circ} \mathrm{C}$ with $20 \mathrm{U}$ HinfI restriction enzyme (Gibco-BRL) in the presence of $4 \mathrm{~mm}$ spermidine. The DNA fragments were separated by electrophoresis over $24 \mathrm{~h}$ on $20 \times 20 \mathrm{~cm} 0.6$ per cent agarose gels in TBE buffer $(0.09 \mathrm{M}$ Tris, $0.09 \mathrm{M}$ boric acid, $0.002 \mathrm{M}$ EDTA). The DNA was transferred in $10 \times$ SSC to nylon membranes (Hybond $\mathrm{N}^{+}$, Amersham) by Southern blotting. SLPs were $\left[\alpha{ }^{32} \mathrm{P}\right] \mathrm{dCTP}$-labelled using a multiprime labelling kit (Gibco-BRL). SLPs were hybridized to the filters overnight at $68^{\circ} \mathrm{C}$ in a $0.5 \mathrm{M}$ Na phosphate $\mathrm{pH} 7.2,1 \mathrm{~mm}$ EDTA and 7 per cent (w/v) SDS buffer (Church \& Gilbert, 1984) with variable amounts of human competitor DNA. High stringency washes at $68^{\circ} \mathrm{C}$ were performed up to two times in $0.1 \times$ SSC, 0.1 per cent SDS for $15 \mathrm{~min}$. Autoradiography was performed overnight at $-70^{\circ} \mathrm{C}$ using two Kodak regular intensifying screens and Kodak X-OMAT K or X-OMAT AR films.

\section{Allele designation}

The alleles of the three minisatellite markers behave as codominant markers and were observed to segregate in a Mendelian fashion in blue tit families (Kempenaers et al., 1992). Allele sizes were determined by comparing the migration distances of the bands in the gel to those of length markers of known sizes (Analytical Marker System, Promega). These distances were converted into allele sizes using the method of Duggleby et al. (1981). Because of the limited resolution of the agarose gel electrophoresis technique, resulting in quasi-continuous allele distributions, alleles were grouped in 100 bp classes.

\section{Computer software}

Statistical tests of Hardy-Weinberg (HW) equilibrium and of population differentiation were performed using the GENEPOP computer program (Raymond \& Rousset (1995a,b)). This software package computes exact tests for deviations from Hardy-Weinberg equilibrium, genotypic disequilibrium and population differentiation. The program FSTAT (Goudet, 1994) was used to estimate Wright's $F$-statistics. The fixation index $F_{\mathrm{ST}}$ is a measure of the genetic differentiation between subpopulations. 
$F_{\text {IS }}$ and $F_{\text {IT }}$ are measures of the deviation from HW proportions within subpopulations and in the total population respectively. $F_{\text {IT }}$ takes into account the effects of random genetic differentiation between subpopulations $\left(F_{\mathrm{ST}}\right)$ and the effects of deviation from HW equilibrium in subpopulations $\left(F_{\text {IS }}\right)$. Because of the nature of the theta estimator (Weir, 1990 ), negative $F_{\mathrm{ST}}$ values can occur. Using $F_{\mathrm{ST}}$, the number of migrants $(\mathrm{Nm})$ between populations was estimated by

$N m=\left(1 / F_{\text {st }}-1\right) / 4$.

The model behind this formula assumes neutral markers and an island population structure. As an alternative to $F_{\mathrm{ST}}$, Nei's genetic distance measure, provided by the PHYLIP version $3.5 \mathrm{c}$ software package (Felsenstein, 1989), was calculated.

\section{Results}

\section{Allelic polymorphism}

Table 1 illustrates the extreme variability displayed by the three minisatellite loci studied. The observed heterozygosities range from 79 per cent to 97 per cent, and the number of alleles is high for each locus (mean number of alleles per breeding season: for $\mathrm{MS} 1=13.2$; for $\mathrm{MS3}=24$; for $\mathrm{MS} 14=31.2$ ). For each marker a positive correlation was found between the number of alleles detected and the

Table 1 Variability displayed by three minisatellite SLPs in the blue tit

\begin{tabular}{|c|c|c|c|c|c|c|}
\hline Population & $H_{\text {obs }}$ & $H_{\text {exp }}$ & $F_{\text {obs }}$ & $F_{\text {exp }}$ & $N_{I}$ & $N_{\mathrm{A}}$ \\
\hline \multicolumn{7}{|l|}{ MS1 } \\
\hline CX 92 & 0.85 & 0.88 & 9 & 7.37 & 59 & 11 \\
\hline CX 93 & 0.84 & 0.79 & 4 & 5.34 & 25 & 14 \\
\hline $\mathrm{CX} \mathrm{T}$ & 0.87 & 0.88 & 11 & 10.26 & 84 & 14 \\
\hline PB 92 & 0.97 & 0.89 & 1 & 4.37 & 39 & 15 \\
\hline PB 93 & 0.79 & 0.88 & 6 & 3.45 & 29 & 12 \\
\hline PB T & 0.90 & 0.89 & 7 & 7.36 & 68 & 16 \\
\hline BO 92 & 0.87 & 0.87 & 4 & 3.78 & 30 & 11 \\
\hline BO 93 & 0.85 & 0.89 & 10 & 7.59 & 67 & 16 \\
\hline BO $\mathrm{T}$ & 0.86 & 0.89 & 14 & 11.14 & 97 & 17 \\
\hline \multicolumn{7}{|l|}{ MS3 } \\
\hline CX 92 & 0.97 & 0.95 & 2 & 3.25 & 63 & 26 \\
\hline CX 93 & 0.92 & 0.92 & 2 & 2.03 & 25 & 24 \\
\hline $\mathrm{CX} \mathrm{T}$ & 0.95 & 0.95 & 4 & 4.36 & 88 & 30 \\
\hline PB 92 & 0.90 & 0.93 & 4 & 2.63 & 39 & 25 \\
\hline PB 93 & 0.86 & 0.92 & 4 & 2.47 & 29 & 23 \\
\hline PB T & 0.88 & 0.94 & 8 & 4.16 & 68 & 28 \\
\hline BO 92 & 0.97 & 0.93 & 1 & 2.07 & 29 & 21 \\
\hline BO 93 & 0.88 & 0.94 & 8 & 4.18 & 66 & 25 \\
\hline $\mathrm{BO} \mathrm{T}$ & 0.91 & 0.94 & 9 & 5.82 & 95 & 28 \\
\hline \multicolumn{7}{|l|}{ MS14 } \\
\hline CX 92 & 0.89 & 0.96 & 9 & 3.60 & 71 & 37 \\
\hline CX 93 & 0.96 & 0.91 & 1 & 2.18 & 25 & 30 \\
\hline $\mathrm{CX} \mathrm{T}$ & 0.91 & 0.95 & 9 & 4.43 & 96 & 33 \\
\hline PB 92 & 0.96 & 0.95 & 2 & 1.73 & 39 & 31 \\
\hline PB 93 & 0.97 & 0.94 & 1 & 1.64 & 29 & 26 \\
\hline PB T & 0.96 & 0.96 & 3 & 3.04 & 68 & 38 \\
\hline BO 92 & 0.95 & 0.97 & 1 & 1.62 & 30 & 27 \\
\hline BO 93 & 0.96 & 0.91 & 6 & 3.00 & 69 & 36 \\
\hline $\mathrm{BO} \mathrm{T}$ & 0.93 & 0.96 & 7 & 4.23 & 99 & 39 \\
\hline
\end{tabular}

Breeding seasons 1992, 1993 and $\mathrm{T}=$ combined data of 1992 and 1993.

$H_{\text {obs}}$, observed heterozygosity; $H_{\text {exp }}$, expected heterozygosity, assuming HardyWeinberg equilibrium; $F_{\text {obs }}$, observed number of homozygous individuals; $F_{\text {exp }}$, expected number of homozygous individuals; $N_{\mathrm{l}}$, number of individuals sampled; $N_{\mathrm{A}}$, number of alleles. 
number of individuals sampled (Spearman rank correlation coefficient; MS1: $r=0.41, \quad P=0.57$, d.f. $=4$; MS3: $r=0.88, P=0.021$, d.f. $=4$; MS14: $r=0.94, P=0.006$, d.f. $=4)$. Because of the large differences in sample sizes and the highly polymorphic nature of these markers, resulting in an incomplete description of allele frequencies, we pooled the data from the different breeding seasons (making sure not to include the same individual twice). Pooling the data from different breeding seasons seems justified, because the populations are not significantly differentiated between the two successive breeding seasons (Table 2 ).

\section{Hardy-Weinberg equilibrium}

Table 3 shows that Hardy-Weinberg equilibrium does not always hold in these populations. Only MS1 never shows significant deviations from HW expectations. MS3 appears to be the least stable locus. In the $\mathrm{BO}$ population a consistent deficit in heterozygotes is shown for the three markers. This could be the result of clumping the data from several subpopulations in different woodlots. This inbreeding-like effect of sampling from a subdivided population is called the Wahlund effect (Hartl \& Clark, 1989). The differences between observed and

Table 2 Tests for differentiation of a blue tit population between the successive breeding seasons 1992 and 1993

\begin{tabular}{llrrr}
\hline Population & Marker & \multicolumn{1}{c}{$F_{\text {ST }}$} & $P$ & SE \\
\hline PB & cPcaMS1 & 0.000 & 0.1859 & 0.0010 \\
PB & cPcaMS3 & 0.015 & 0.0593 & 0.0006 \\
PB & cPcaMS14 & -0.004 & 0.6868 & 0.0014 \\
PB & Combined & 0.004 & 0.1348 & \\
BO & cPcaMS1 & -0.006 & 0.7976 & 0.0009 \\
BO & cPcaMS3 & -0.001 & 0.1287 & 0.0009 \\
BO & cPcaMS14 & -0.003 & 0.7502 & 0.0014 \\
BO & Combined & -0.003 & 0.5276 & \\
CX & cPcaMS1 & 0.009 & 0.1023 & 0.0008 \\
CX & cPcaMS3 & -0.003 & 0.4882 & 0.0015 \\
CX & cPcaMS14 & 0.003 & 0.1725 & 0.0013 \\
CX & Combined & 0.003 & 0.1469 & \\
\hline
\end{tabular}

$F_{\mathrm{ST}}$, fixation index; $P$, exact probability; $\mathrm{SE}$, standard error.

Table 3 Probabilities (and standard errors) for deviation from Hardy-Weinberg equilibrium for each marker for the combined data from blue tit populations

\begin{tabular}{llccrc}
\hline Population & Marker & $P$ & SE & \multicolumn{1}{c}{$F_{\text {IS }}$} & Het. \\
\hline PB & cPcaMS1 & 0.5358 & 0.0041 & 0.001 & Excess \\
PB & cPcaMS3 & $\mathbf{0 . 0 0 5 1}$ & 0.0009 & $\mathbf{0 . 0 6 8}$ & Deficit \\
PB & cPcaMS14 & 0.3657 & 0.0108 & 0.007 & Excess \\
PB & Combined & $\mathbf{0 . 0 3 1 6}$ & & 0.026 & \\
BO & cPcaMS1 & 0.0584 & 0.0019 & 0.038 & Deficit \\
BO & cPcaMS3 & $\mathbf{0 . 0 0 0 7}$ & 0.0004 & 0.041 & Deficit \\
BO & cPcaMS14 & 0.3891 & 0.0101 & 0.034 & Deficit \\
BO & Combined & $\mathbf{0 . 0 0 1 2}$ & & $\mathbf{0 . 0 3 8}$ & \\
CX & cPcaMS1 & 0.1396 & 0.0023 & 0.019 & Deficit \\
CX & cPcaMS3 & 0.1634 & 0.0056 & -0.004 & Excess \\
CX & cPcaMS14 & $\mathbf{0 . 0 0 0 1}$ & 0.0001 & $\mathbf{0 . 0 4 7}$ & Deficit \\
CX & Combined & $\mathbf{0 . 0 0 0 2}$ & & 0.021 & \\
\hline
\end{tabular}

Het., the observed excess or deficit of heterozygote individuals compared to the expected number.

Significant results are marked bold.

Bold inbreeding coefficients ( $F_{\text {Is }}$ values) are significant at $P<0.05$. 
expected numbers of homozygotes are not significant $\left(\chi_{6}^{2}=12.07, P>0.05 \mathrm{NS}\right.$, for the combined data of the markers). However, $F_{\text {IS }}$ for the combined data is significantly different from 0 . The subpopulations of plot BO are not significantly differentiated (Table 4) and the consistent deficiency in heterozygotes disappears when considering the subpopulations separately (data not shown).

\section{Population differentiation and genetic distance}

Table 4 displays the overall differentiation (exact test, Raymond \& Rousset, 1995a) between all populations. All markers indicate significant differentiation. By combining the results of the three markers (Fisher's method), highly significant results are obtained, confirming differentiation between the populations. The BO subpopulations, however, are not significantly differentiated (Table 4). Table 5 gives the differentiation between all combinations of two populations using the exact test for population differentiation. Many of the markers are significantly differentiated between populations, and the combined results of the three markers again indicate differentiation. The probabilities given in Table 4 are not indicative of the degree of differentiation between the populations. Therefore, Wright's $F$ statistics (for all three markers) were estimated according to Weir using the program FSTAT (Weir, 1990; Table 6 gives the total $F$-statistics of the three markers combined). All $F_{\mathrm{ST}}$ values were shown to be significantly different from zero, except between the subplots of $\mathrm{BO}$, confirming the results obtained by the exact test. Table 6 also includes estimates of the number of migrants $(\mathrm{Nm})$ among the populations. These numbers clearly show that gene flow is high among all populations. All total $F_{\text {IS }}$ values in Table 6,

Table 4 Overall test of differentiation between blue tit populations $\mathrm{PB}, \mathrm{CX}$ and $\mathrm{BO}$ and between $\mathrm{BO}$ subpopulations LA/VS, ZZ and KB

\begin{tabular}{llcc}
\hline Locus & Populations & $P$ & SE \\
\hline cPcaMS1 & PB/CX/BO & $\mathbf{0 . 0 0 0 0 3}$ & 0.00001 \\
cPcaMS3 & PB/CX/BO & $\mathbf{0 . 0 0 0 0 0}$ & 0.00000 \\
cPcaMS14 & PB/CX/BO & $\mathbf{0 . 0 2 3 5 3}$ & 0.00071 \\
Combined & PB/CX/BO & $\mathbf{0 . 0 0 0 0 1}$ & \\
cPcaMS1 & BO & 0.20100 & 0.00138 \\
cPcaMS3 & BO & 0.05938 & 0.00084 \\
cPcaMS14 & BO & 0.36667 & 0.00223 \\
Combined & BO & 0.09271 & \\
\hline
\end{tabular}

Significant results are indicated in bold. except that for populations CX and PB, are significantly different from zero, indicating deviations from Hardy-Weinberg equilibrium in the populations. When considering the $F_{\text {Is }}$ values of the separate loci in the separate populations (Table 3 ), only two significant deviations were obtained, not always confirming the exact test on HW equilibrium.

Nei's genetic distance measure yields the smallest genetic distance $(0.1641)$ for the two populations that are the closest to one another, i.e. PB and CX (separated by $2 \mathrm{~km}$ ), while genetic distances are higher between these populations and the more geographical distant $\mathrm{BO}$ population (0.1731 for $\mathrm{CX} / \mathrm{BO}$ separated by $13 \mathrm{~km}$; and 0.1781 for $\mathrm{PB} / \mathrm{BO}$, separated by $15 \mathrm{~km}$ ).

Table 5 Tests of differentiation between pairs of blue tit populations

\begin{tabular}{|c|c|c|c|}
\hline Locus & Population & $P$ & SE \\
\hline cPcaMS1 & $\mathrm{PB} / \mathrm{BO}$ & 0.02701 & 0.00053 \\
\hline сPсaMS3 & $\mathrm{PB} / \mathrm{BO}$ & 0.00008 & 0.00002 \\
\hline cPcaMS14 & $\mathrm{PB} / \mathrm{BO}$ & 0.45807 & 0.00224 \\
\hline Combined & $\mathrm{PB} / \mathrm{BO}$ & $<0.00010$ & \\
\hline cPcaMS1 & $\mathrm{PB} / \mathrm{CX}$ & 0.00555 & 0.00023 \\
\hline cPcaMS3 & $\mathrm{PB} / \mathrm{CX}$ & 0.01518 & 0.00033 \\
\hline сPсaMS14 & $\mathrm{PB} / \mathrm{CX}$ & 0.19233 & 0.00173 \\
\hline Combined & $\mathrm{PB} / \mathrm{CX}$ & $<0.00500$ & \\
\hline cPcaMS1 & $\mathrm{BO} / \mathrm{CX}$ & 0.00059 & 0.00007 \\
\hline сPcaMS3 & $\mathrm{BO} / \mathrm{CX}$ & 0.00042 & 0.00004 \\
\hline cPcaMS14 & $\mathrm{BO} / \mathrm{CX}$ & 0.00174 & 0.00011 \\
\hline Combined & $\mathrm{BO} / \mathrm{CX}$ & $<0.00010$ & \\
\hline
\end{tabular}

Significant results are indicated in bold.

Table 6 Total $F$-statistics (standard error) for blue tit populations using the three genetic markers combined

\begin{tabular}{lcccc}
\hline Population & $F_{\text {IT }}$ & $F_{\text {ST }}$ & $F_{\text {IS }}$ & $N m$ \\
\hline CX/BO/PB & $\mathbf{0 . 0 3 4}$ & $\mathbf{0 . 0 0 6}$ & $\mathbf{0 . 0 2 8}$ & 39 \\
& $(0.003)$ & $(0.002)$ & $(0.003)$ & \\
CX/BO & $\mathbf{0 . 0 3 5}$ & $\mathbf{0 . 0 0 7}$ & $\mathbf{0 . 0 2 9}$ & 35 \\
& $(0.005)$ & $(0.002)$ & $(0.003)$ & \\
CX/PB & 0.020 & $\mathbf{0 . 0 0 7}$ & 0.014 & 48 \\
& $(0.011)$ & $(0.004)$ & $(0.014)$ & \\
PB/BO & $\mathbf{0 . 0 3 9}$ & $\mathbf{0 . 0 0 6}$ & $\mathbf{0 . 0 3 3}$ & 41 \\
& $(0.013)$ & $(0.004)$ & $(0.010)$ & \\
BO & $\mathbf{0 . 0 5 8}$ & 0.002 & $\mathbf{0 . 0 5 6}$ & 131 \\
& $(0.006)$ & $(0.0006)$ & $(0.006)$ & \\
\hline
\end{tabular}

Bold indicates values significantly different from zero $(P<0.05)$.

$\mathrm{Nm}$ estimated number of migrants among the populations. 


\section{Discussion and conclusion}

In all populations, all markers displayed an extremely high heterozygosity. This is typical of this type of genetic marker (Jeffreys et al., 1988). An exact test for Hardy-Weinberg equilibrium showed that MS3 was in disequilibrium in two populations and that MS14 was in disequilibrium in plot CX. The reasons for the departures from HW equilibrium are not clear. The markers are assumed to behave neutrally (Clark 1987; Jeffreys et al., 1988). The most likely explanations would therefore be dispersal among populations (which is high in blue tits (Berndt \& Sternberg, 1969), and the relatively high variance in reproductive success between blue tits (Dhondt, 1989, Kempenaers et al., 1992). Random genetic drift could be important because of the large number of alleles and the relatively small population sizes.

Exact tests on differentiation show that all populations are significantly differentiated from each other. These results were confirmed by Wright's $F_{\mathrm{ST}}$ values which all differed significantly from zero. This was very surprising given the short distance between the populations and the ability of blue tits to disperse. No differentiation, however, was found between the subpopulations of the $\mathrm{BO}$ population which are separated by no more than $0.5-2 \mathrm{~km}$, indicating sufficiently high levels of gene flow to prevent differentiation. Most $F_{\text {IS }}$ indices for the separate loci (Table 3), indicating the amount of inbreeding in the populations, were very low. Moreover, there was no consistent heterozygote deficiency except for the total BO population, as should be the case with inbreeding. Within the BO population, our data suggest the occurrence of a Wahlund effect, with a consistent heterozygote deficit in the overall population but not in the subpopulations.

When calculating the number of migrants $(\mathrm{Nm})$ from the $F_{\text {ST }}$ values, it becomes clear that gene flow is very high among all blue tit populations. These values are comparable to those obtained in most other avian studies (Rockwell \& Barrowclough, 1987; Johnson \& Marten, 1989) and should only be considered as being very high. Despite the high $\mathrm{Nm}$ values and the small distances between populations, significant differentiation still occurs for these minisatellite markers. It therefore appears that random drift in combination with relatively high mutation rates (maximal mutation rates: 0.0024 for cPcaMS1, 0.0032 for $\mathrm{c} P c a \mathrm{MS} 3$ and 0.001 for cPcaMS14; Verheyen et al., 1994) and variances in reproductive success (which result in a reduction of the effective population size), have an important influence on hypervariable loci. Furthermore, their effects can be high enough to prevent gene flow from equalizing the allele distributions of nearby populations. Hence, gene flow may have to be much higher to prevent substantial differentiation at these loci. Measuring $F_{\text {ST }}$ between pairs of populations indicates that the neighbouring plots $\mathrm{CX}$ and $\mathrm{PB}$ are the least differentiated. This isolation by distance effect can also be seen by considering Nei's genetic distance measure between the populations. However, a more extensive study, including some more distant populations, should be performed to verify whether these markers are indeed able to detect isolation by distance or whether the differentiation between populations is generated solely by chance processes.

In conclusion, we can say that these markers are not representative of the genome because of their general characteristics. The high mutation rates and the high number of alleles (resulting in the need for large sample sizes) can present problems in studying population processes. However, in this paper we have shown that they could be informative in population studies, because they are most probably selectively neutral and because they allow us to detect small differences (possibly reflecting random drift) between very nearby populations despite high levels of gene flow. These markers might therefore be useful in estimating levels of gene flow between populations. However, more elaborate studies will have to be undertaken to confirm this.

\section{Acknowledgements}

We thank Michel Raymond for providing the GENEPOP software, and J. Goudet for providing FSTAT. We also thank Stefan Van Dongen, Francisco Pulido and Professor van Noordwijk for reading and giving helpful comments on the manuscript. We thank the Bracht family for allowing us to work on their estate. G.R.V. holds an Instituut voor Wetenschappelijk Onderzoek in Nijverheid en Landbouw grant, B.K. is Research Assistant of the Belgian National Fund for Scientific Research and C.V.B. and E.M. are Research Associates of the Belgian National Fund for Scientific Research. This study was supported by grant FKFO 2.0063.93 from the Belgian National Foundation for Scientific Research to A.D.

\section{References}

BALL, M. R., FREEMAN, S., JAMES, F. C., BERMINGHAM, E. AND AVISE, J. C. 1988. Phylogeographic population structure of red-winged blackbirds assessed by mito- 
chondrial DNA. Proc. Natl. Acad. Sci., U.S.A., 85, $1558-1562$.

BERNDT, R. AND STERNBERG, H. 1969. Terms, studies and experiments on the problems of bird dispersion. Ibis, 110, 256-269.

BURKE, T. AND BRUFORD, M. w. 1987. DNA Fingerprinting in birds. Nature, 327, 149-152.

CHURCH, G. M. AND GILBERT, w. 1984. Genomic sequencing. Proc. Natl. Acad. Sci. U.S.A., 81, 1991-1995.

CLARK, A. G. 1987. Neutrality tests of highly polymorphic restriction fragment length polymorphisms. Am. J. Hum. Genet., 41, 948-956.

DHONDT, A. A. 1989. Blue tit. In: Newton, I. A. (ed.) Lifetime Reproduction in Birds, pp. 15-33. Academic Press, New York.

DUGGLEBY, R. G., KINNS, H. AND ROOD, J. I. 1981. A computer program for determining the size of DNA restriction fragments. Analyt. Biochem., 110, 723-725.

FELSENSTEIN, J. 1989. PHYLIP-Phylogeny inference package (version 3.2). Cladistics, 5, 164-166.

GILBERT, D. A., LEHMAN, N., O'BRIEN, S. J. AND WAYNE, R. K. 1990. Genetic fingerprinting reflects population differentiation in the California Channel Island fox. Nature, 344, 764-766.

GOUDET, J. 1994. FSTAT, a program for IBM PC compatibles to calculate Weir and Cockerham's estimators of F-statistics. Institut de Zoologie et d'Ecologie Animale, Université de Lausanne, Switzerland.

HAIG, S. M., BELTHOFF, J.R. AND ALLEN, D.H. 1993. Examination of population structure in red-cockaded woodpeckers using DNA profiles. Evolution, 47, 185-194.

HANOTTE, O., BURKE, T., ARMOUR, J. A. L. AND JEFFREYS, A. J. 1991. Hypervariable minisatellite DNA sequences in the Indian Peafowl Pavo cristatus. Genomics, 9, 587-597.

haRTL, D. L. AND Clark, A. G. 1989. Principles of Population Genetics. Sinauer Associates, Sunderland, MA.

JEFFREYS, A. J., WILSON, V. AND THEIN, S. L. 1985. Individual-specific 'fingerprints' of human DNA. Nature, 316, 76-79.

JEFFREYS, A. J., WILSON, V., THEIN, S. L., WEATHERALL, D. J. AND PONDER, B. A. J. 1986. DNA "fingerprints" and segregation analysis of multiple markers in human pedigrees. Am. J. Hum. Genet., 39, 11-24.

JEFFREYS, A. J., ROYLE, N, J., WILSON, V. AND WONG, $z$. 1988. Spontaneous mutation rates to new length alleles at tandem repetitive hypervariable loci in human DNA. Nature, 332, 278-281.
JOHNSON, N. K. AND MARTEN, J. A. 1989. Evolutionary genetics of flycatchers. III. Variation in Empidonax hammondii (Aves: Tyrannidae). Can. J. Zool., 69, 232-238.

KEMPENAERS, B., VERHEYEN, G. R., VAN DEN BROECK M., BURKE T., VAN BROECKHOVEN, C. AND DHONDT, A. A. 1992. Extra-pair paternity results from female preference for high-quality males in the blue tit. Nature, 357, 494-496.

KEMPENAERS, B., VERHEYEN, G. R. AND DHONDT, A. A. 1995. Mate guarding and copulation behaviour in monogamous and polygynous blue tits: do males follow a bestof-a-bad-job strategy? Behav. Ecol. Sociobiol., 36, $33-42$.

LEBERG, P. L. 1990. Influence of fragmentation and bottlenecks on genetic divergence of wild turkey populations. Conserv. Biol., 5, 522-530.

MOORE, w. S., GRAHAM, J. H. AND PRICE, J. T. 1991. Mitochondrial DNA variation in the Northern Flicker (Colaptes auratus, Aves). Mol. Biol. Evol., 8, 327-344.

RAYMOND, M. AND ROUSSET, F. 1995a. An exact test for population differentiation. Evolution, (in press).

RAYMOND, M. AND ROUSSET, F. 1995b. GENEPOP (ver. 1.2): a population genetics software for exact test and ecuniscism. J. Hered., 95, 248-249.

ROCKWELL, R. F. AND BARROWCLOUGH, G. F. 1987. Gene flow and the genetic structure of populations. In: Cooke, F. and Buckley, P. A. (eds) Avian Genetics: a Population and Ecological Approach, pp. 223-255. Academic Press, New York.

STANGEL, P. W., LENNARTZ, M. R. AND SMITH, M. H. 1992. Genetic variation and population structure of red-cockaded woodpeckers. Conserv. Biol., 6, 283-292.

TABERLET, P., MEYER, A. AND BOUVET, J. 1992. Unusual Mitochondrial DNA polymorphism in two local populations of blue tit Parus caeruleus. Mol. Ecol., 1, 27-36.

VERHEYEN, G. R., KEMPENAERS, B., BURKE, T., VAN DEN BROECK, M., VAN BROECKHOVEN, C. AND DHONDT, A. A. 1994. Identification of hypervariable single locus minisatellite DNA probes for the blue tit (Parus caeruleus). Mol. Ecol., 3., 137-143.

wEIR, B. S. 1990 . Genetic Data Analysis. Sinauer Associates, Sunderland, MA.

WENINK, P. W., BAKER, A. J. AND TILANUS, M. G. J. 1993. Hypervariable-control-region sequences reveal global population structuring in a long-distance migrant shorebird, the Dunlin (Calidris alpina). Proc. Natl. Acad. Sci., U.S.A., 90, 94-98 